\title{
Multifactorial Optimization Approach for Determination of Polycyclic Aromatic Hydrocarbons in Sea Sediments of Turkish Mediterranean Coast
}

\author{
Semra G. Tuncel*, Tansel Topal \\ Middle East Technical University, Chemistry Department, Ankara, Turkey \\ E-mail: *semratun@metu.edu.tr \\ Received April 20, 2011; revised June 17, 2011; accepted July 1, 2011
}

\begin{abstract}
Present study aims the optimisation and validation of the extraction procedures for the determination of polycyclic aromatic hydrocarbons (PAHs) in sediment samples. As analytical techniques, gas chromatographymass detector (GC-MS) and gas chromatography-flame ionization detector (GC-FID). The optimized methods were soxhlet extraction, ultrasonic bath extraction and solid phase micro extraction (SPME). The factorial design was used in order to search out the main factors affecting extraction efficiencies. The best extraction method was chosen as ultrasonic bath extraction and optimum values for main factors were selected for the development of this extraction method. The optimised methods were validated to confirm the reliability. Percent errors were in between $1.40 \%-25.0 \%$. Relative standard deviation was less than $1 \%$ and limit of detection changed from 0.006 to $0.028 \mathrm{mg} / \mathrm{L}$. The accuracy of the method was verified by analyzing National Institute of Standards and Technology, Standard Reference Material 1597a (NIST SRM 1597a). The selectivity, accuracy and precision were quite adequate for the determination of PAHs in sediment samples. The best extraction and analysis methods were then applied to determine 16 PAHs in sea sediments from Ölüdeniz Lagoon, of Turkish Meditreanean coast and marine sediment, SRM 1941b. The highest observed cocentration for PAHs was $0.620 \mathrm{mg} \cdot \mathrm{kg}^{-1}$ for Acenaphtene.Total sum of the concentrations for all the observed PAHs was $1.854 \mathrm{mg} \cdot \mathrm{kg}^{-1}$.
\end{abstract}

Keywords: PAH, Sediment, Response Surface Methodology, Pareto Chart, Chemometrics

\section{Introduction}

Polycyclic aromatic hydrocarbons (PAHs) which are commonly found in the different compartment of the environment including drinking water, seawater, aerosols, soil and sediments. Since they are carcinogenic and commonly found around us, they attracted more attention around the world. They enter to aquatic environments by oil spills, industrial discharges, municipal and urban runoff and atmospheric precipitation. As PAHs are hydrophobic, they partition to sediments through absorption by organic matter in the sediments. Therefore it is important to investigate sediment in order to monitor PAH concentration in aquatic environment, and it is also important to develop, validate and optimize methodologies for the determination of PAH level in sediments [1].

There are a lot of studies related with PAH analysis and method validation in sediment samples [2-7]. It is very well known that extraction is the first and important step in the determination of PAHs especially in solid matrices like sediments. Extraction methods for determination of PAHs from sediments were validated and modified in some studies [3]. The mostly used methods for extracting of PAHs from sediments and other solid environmental materials are mechanical shaking, soxhlet extraction, ultrasonic extraction, solid phase extraction (SPE) and solid phase micro extraction (SPME). There are several important factors affecting efficiency of the extraction of PAHs from sediment samples, like solvent, sorbent and clean up procedures. It is important to say that there is no single methodology to provide very good results for at least EPA's priority 16 PAHs analysis. Some investigators obtained lower detection limits but only four or five selected PAHs [3]; therefore there is a need to investigate different extraction methodologies and their optimization for obtaining better extraction 
efficiencies of PAHs in sediment samples. In most optimization studies generally only one or two sediment extraction methods were tried to be optimized without using a chemometric approach [8-10] In this study, three extraction methods were compared and one of them (solid phase micro extraction) is very novel. Apart from the other studies; both analysis methods (GC-FID, GCMS) and extraction methods were optimized before starting PAH analysis in sediment samples. Most studies optimized either extraction method or analysis method alone [3,4] not both. For the optimization of extraction methods, chemometrics [5]. "A new approach" was utilized and instead of using established methods. The most important advantage of chemometrics, is the minimisation of the number of required experiments which in turn results in the reduction of reagent consumption and labor. Therefore chemometrics is faster and more cost effective than traditional one variable at a time technique. By using this method it is possible to study several control factors simultaneously. It is also possible to develop mathematical models providing determination of the relevance, statistical significance of factors and the estimation of interaction effects between factors [6]. As an analytical technique, capillary gas chromatography (GC) coupled to either flame ionization (FID) or mass spectrometry (MS) detectors were used. By this way both analysis and extraction methods were optimised and validated for the PAH analysis in sediment samples. The developed methods were tested on SRM 1941b and applied to real samples.

The aims of this study were (i) to develop analysis methodologies for the analysis of PAHs in sea sediments collected in Ölüdeniz Lagoon by using GC-FID and GC-MS (ii) to optimize extraction methods including soxhlet, ultrasonic and solid phase micro extraction (SPME) (iii) to evaluate the significance of the factors and interactions affecting extraction efficiency.

\section{Experimental}

\subsection{Reagents and Materials}

All chemicals used during the study were of analytical reagent grade. Two different PAH standards; Restek (ISO 9001 registered in methylene chloride, $2000 \mathrm{ug} / \mathrm{mL}$ ) and Environmental Protection Agency (EPA) 610 (in methanol: methylene chloride having a range of 10-1000 ppm, Supelco) were used. Standards (except for SPME) for the calibration curve were prepared diluting them with dichloromethane (DCM, Merck, HPLC-grade). Standard reference materials which were SRM 1941b (marine sediment) and 1597a (complex mixture of polycyclic aromatic hydrocarbons from coal tar prepared in
DCM), were bought from National Institute of Standards and Technology. The surrogate solution (Surrogate Standard Base/Neutrals Mix 19) including three surrogates; nitrobenzene D5, p-terphenyl D14, 2-fluorobiphenyl was bought from Dr. Ehrenstorfer. The concentration of surrogate was $1000 \mu \mathrm{g} \cdot \mathrm{ml}^{-1}$ in DCM solution. The GCMS surrogate solution mix which includes AcenaphteneD10 (Ace-D10), Phenanthrene-D10 (Phe-D10), Chrysene-D12 (Chr-D12) and Perylene-D12 (Per-D12) (1000 $\mathrm{mg} / \mathrm{L}$, in Acetone) was bought also from Dr. Ehrenstorfer. High purity nitrogen (99.999), helium (99.999), hydrogen (99.999) and dry air (99.999) were used for the analyses. The sodium sulphate $\left(\mathrm{Na}_{2} \mathrm{SO}_{4}\right.$, Merck, Darmstadt, Germany) was used for the removal of the water from extracted samples. High purity glass wool was used during the extraction of sediment samples. Cellulose extraction thimbles (Schleicher \& Schuell Micro science $603,33 \times 80 \mathrm{~mm}$, ref. no. 10350240 ) were used for soxhlet apparatus. Sodium sulphate (anhydrous extra pure, Merck) and glass-wool were pre-cleaned by transferring them to a large glass column (1 L capacity or larger) and washing sequentially with hexane and DCM before use. Sodium sulphate was activated in an oven at $400^{\circ} \mathrm{C}$ for $4 \mathrm{~h}$. Glass-wool was conditioned overnight at $225^{\circ} \mathrm{C}$ in an oven. Cellulose extraction thimbles were cleaned with proper solvents prior to the use with soxhlet extraction system. Alconox detergent powder (Supelco Cat. No. 1104) was used for the cleaning of all glassware used in laboratory. All glasswares were cleaned with detergent in hotwater and rinsed with deionized water. After rinsed with DCM/acetone, they were dried in an oven overnight.

\subsection{Sample Collection and Instrumentation}

Samples (totally 68 sediment samples together with the blank) were collected in 20-22 March, 2003 from Oludeniz Lagoon which is located on the intersection of coastal lines of Mediterranean Sea and Aegean Sea in Turkey. Among the analysed sediment samples 3 of them was collected from the inside and 2 of them was collected from outside of the Lagoon.

Before the extraction, sediment samples were homogenized and used for the optimization of the extraction methods. For doing this 100 gram portions were taken from each sample, put in a bottle having a capacity of 5.0 $\mathrm{L}$ and mixed. The prepared sample was kept in refrigerator at $+4^{\circ} \mathrm{C}$. The samples were mixed with sodium sulphate prior to extraction with soxhlet or ultrasonic bath methods. Following the homogenization water content of the samples was determined. Moisture content of the samples was ranged from $3 \%-4 \%$.

All the PAH analysis was performed with HewlettPackard (HP) 6890 GC system equipped with a flame 
ionization detector. The system has also 5973 mass selective detector. Heidolph rotary evaporator (laborota 4000 efficient) and mini-vap evaporator with 6 ports (Catalog No: 22970, Cat. No: 22971) were used for the evaporation of solvents. Bransonic ultrasonic bath (Model B-2200 E4, 205 W, $220 \mathrm{~V}$ ) was used for the sample extractions. Deionised water (ultra filtered type 1 water) was supplied by Barnstead nanopure ultrapure water system.

\section{Results and Discussion}

Before starting optimization of extraction methods the most proper solvent was selected. In addition some preliminary experiments were done for SPME and best fiber was selected for both direct SPME and HSSPME. Both studies are discussed below.

\subsection{Solvent Selection}

The solvents were selected by considering some factors such as suitability to analytes polarity, suitability to standards solvent, toxicity and cost. In this study, three solvents; DCM (solvents of the standards), acetone (least toxic solvent) and hexane (suitable to polarity of analytes) were selected under the EPA guidance. The most proper (which extracts better than the others) three solvents/ solvent pairs were chosen as hexane/acetone, DCM/ acetone and DCM. Soxhlet extraction was chosen as a method for the solvent selection and weight of sediment, time and solvent volume were $80 \mathrm{~g}, 24 \mathrm{~h}$ and $500 \mathrm{ml}$ respectively. The number of replicate was three. Surrogate was added to sediment sample prior to extraction in order to decide which solvents recovery was the highest after an extraction. According to average surrogate recovery values: DCM/acetone was chosen as best solvent and it was used in both soxhlet and ultrasonic bath extractions (Figure 1).

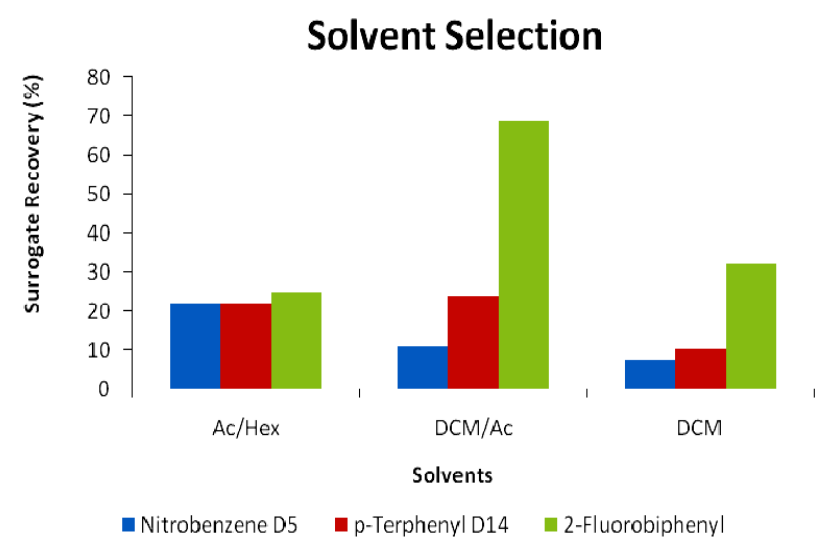

Figure 1. Solvent selection.
Except nitrobenzene D5, other two surrogates, p-terphenyl D14 and 2-fluorobiphenyl gave best result with DCM/acetone. Especially with 2-fluorobiphenyl, DCM/ acetone resulted very good recovery value; $69 \%$. Besides having good recovery value, DCM/acetone was also compatible with the standards solvent (DCM) and it was less toxic than DCM because of the dilution (1:1) with acetone.

\subsection{Fiber Selection for SPME and HSSPME Analysis}

Fiber selection is one of the most important step in SPME and HSSPME extractions. Three kinds of SPME fibers (7, $100 \mu \mathrm{m}$ polydimethylsiloxane (PDMS) and $85 \mu \mathrm{m}$ polyacrylate (PA)) were compared for the extraction efficiency of the 16 EPA priority PAHs by GC-FID. Polyacrylate fiber provided highest extraction efficiency both in direct SPME application and the headspace analysis (HSSPME). In all experiments, acetone was used as a solvent and SRM 1941b (Marine Sediment) was used as a soil matrix.

Peak areas were used for the comparison of three fibers and the selection of the best fiber. Peak areas showed that, $85 \mu \mathrm{m}$ PA had the best efficiency. Extraction efficiencies of the fibers were in the order of; $85 \mu \mathrm{m}$ PA $>$ $100 \mu \mathrm{m}$ PDMS $>7 \mu \mathrm{m}$ PDMS.

\subsection{Optimization of the Instrumentation and Extraction Methodologies}

After the selection of the solvent and the type of the fibers, extraction methodologies were optimised. A chemometric approach was used to reduce the number of experiments. The name of the approach used was the screening design. These type of experiments involve selection of the factors which are important for the success of a process.

Screening analysis $\left(2^{3}\right)$ which contains 8 runs, were designed with the help of statgraphics plus 3.1 program (statistical software) and used for the optimization of extraction methods. Time, amount of sediment and solvent (for ultrasonic bath and soxhlet), adsorption temperature, desorption temperature, adsorption time, desorption time and inlet temperature (for SPME) were evaluated among the factors that may influence the extraction efficiency.

For SPME analysis, representative samples were prepared in $4 \mathrm{ml}$ vials; 8 standard water (prepared from deionized water) solutions containing $1 \mathrm{mg} / \mathrm{L} \mathrm{PAH} \mathrm{and} 2$ $\mathrm{mg} / \mathrm{L}$ surrogate and 8 standard soil samples spiked with $0.5 \mathrm{mg} / \mathrm{kg} \mathrm{PAH}$ and $1 \mathrm{mg} / \mathrm{kg}$ surrogate. These analyses were done with only using the best fiber; $85 \mu \mathrm{m}$ PA. Sediment saples were analyzed with HSSPME and water samples were analyzed with direct SPME. There were 
mainly three factors at two levels (min-max) in direct SPME and HSSPME designs as can be seen from Table 1.

Factor names and levels for soxhlet and ultrasonic bath extractions were also given in Table 1. There were also three factors (A, B and C) and two levels (low and high) in their factorial designs.

As can be seen from Table 1, for soxhlet extraction the most important three factors were; extraction time, solvent volume and amount of sediment. They were investigated at two levels which are low and high. Magnitude of the low and high values were determined by considering EPA procedures and literature studies [11]. Boiling temperature was chosen above the solvents boiling points and it was kept constant during the whole extraction.

For ultrasonic bath extraction, the factors were the same with soxhlet extraction. Extractions were made in room temperature. Low and high values (Table 1) were based on the information given in EPA standards $[12,13]$.

The important three factors; adsorption temperature, inlet temperature and desorption time, for direct SPME, their low and high values were given in Table 2. Adsorption time was kept at maximum level; $60 \mathrm{~min}$ and stayed constant during the optimization. The SPME manual and fibers properties were considered while determining factor levels. For example for proper desorp- tion, inlet temperature cannot be too low or too high. Since equilibrium is reached after a while and values (concentrations, peak areas etc.) stays constant, longer desorption and adsorption times were unnecessary. After an adsorption step, SPME syringe was immediately transferred to the inlet for desorption. Therefore moisture in the needle was neglected.

Table 1 also shows the factors; adsorption temperature, inlet temperature, adsorption time and their levels for HSSPME. Instead of adsorption time in case of SPME optimization, desorption time was kept constant (20 min) during the optimization of HSSPME.

Using screening analyses results which were found in terms of surrogate recoveries (or peak area) pareto charts were drawn. They were used in order to decide the signifance of the main effects. Response surface charts were also prepared by using the same surrogate recovery values. These charts were used to determine optimum values for the selected factors.

The analysis of factorial design results produces main effect and the two-factor interactions pareto charts $(\mathrm{P}=$ 95). In these charts bar lengths are proportional to value of the effect, prividing comparison of the relative importance of effects. Pareto charts also include, the minimum t-values (at the $95 \%$ confidence interval) as a vertical line. When the value of parameter is higher then $\pm t$, it was assumed as a significant [14].

Table 1. Factor levels in factorial design $\left(2^{3}\right)$ for extraction methods.

\begin{tabular}{|c|c|c|c|}
\hline Factor & Key & Low (-) & High (+) \\
\hline \multicolumn{4}{|l|}{ Soxhlet extraction } \\
\hline Time (min) & A & 6 & 24 \\
\hline Solvent volume (ml) & B & 200 & 500 \\
\hline Amount of sediment $(\mathrm{g})$ & $\mathrm{C}$ & 10 & 50 \\
\hline \multicolumn{4}{|l|}{ Ultrasonic bath extraction } \\
\hline Time (min) & A & 15 & 45 \\
\hline Solvent volume (ml) & B & 20 & 50 \\
\hline Amount of sediment (g) & $\mathrm{C}$ & 1 & 10 \\
\hline \multicolumn{4}{|l|}{ Direct SPME } \\
\hline Adsorption temperature $\left({ }^{\circ} \mathrm{C}\right)$ & A & 20 & 80 \\
\hline Inlet temperature $\left({ }^{\circ} \mathrm{C}\right)$ & B & 220 & 280 \\
\hline Desorption time (min) & $\mathrm{C}$ & 5 & 20 \\
\hline \multicolumn{4}{|l|}{ HSSPME } \\
\hline Adsorption temperature $\left({ }^{\circ} \mathrm{C}\right)$ & A & 20 & 80 \\
\hline Inlet temperature $\left({ }^{\circ} \mathrm{C}\right)$ & B & 220 & 280 \\
\hline Adsorption time (min) & $\mathrm{C}$ & 30 & 60 \\
\hline
\end{tabular}


The results of factorial design and pareto charts for each extraction techniques are discussed separately below and given in Figure 2.

\subsection{Pareto Chart Analysis}

Pareto chart for sediment HSSPME analysis was shown in Figure 2(a). As can be seen from the Figure 2(a) no factor was significantly important (below the $\pm \mathrm{t}$ line at $\sim 13, \mathrm{P}=95$ ). However factor A (Adsorption temperature) was relatively more important than the others. The order of the importance of the factors were; adsorption temperature $>$ inlet temperature $>$ adsorption time. Interactions of factors were also shown in the chart. For example the interaction of inlet temperature \& adsorption time was more important (nearly twice as much as important) than the interaction of the adsorption temperature $\&$ inlet temperature and the interaction of the adsorption temperature $\&$ adsorption time. In addition interaction of the adsorption temperature \& inlet temperature and the interaction of the adsorption temperature \& adsorption time had nearly the same importance.

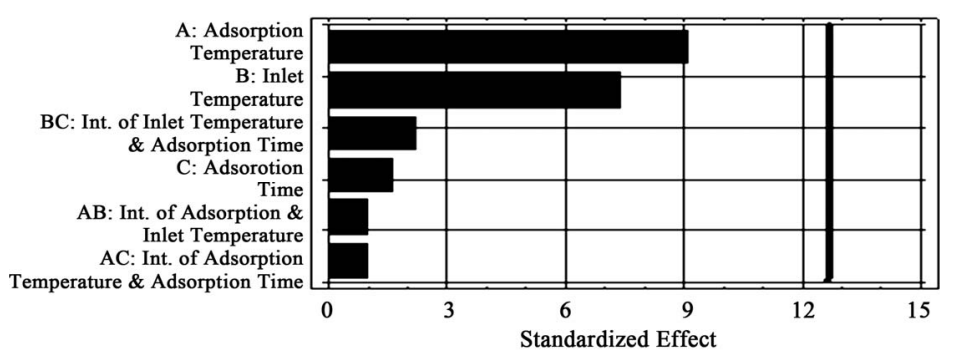

(a)

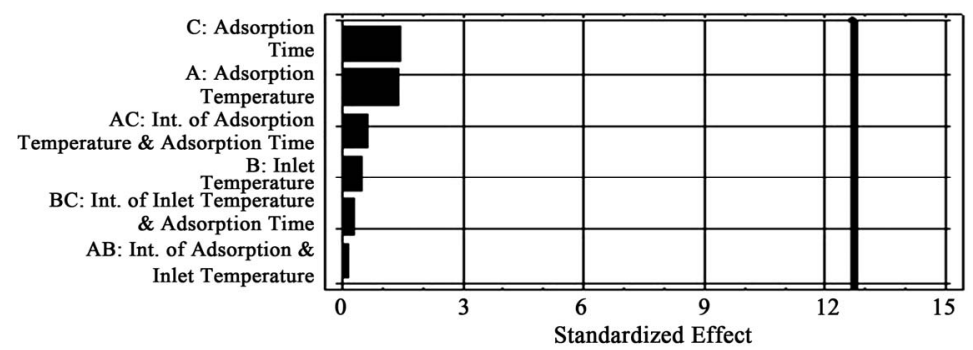

(b)

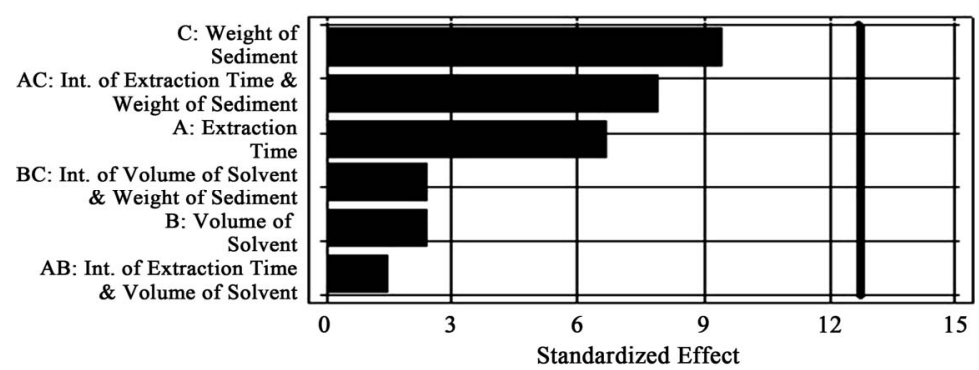

(c)

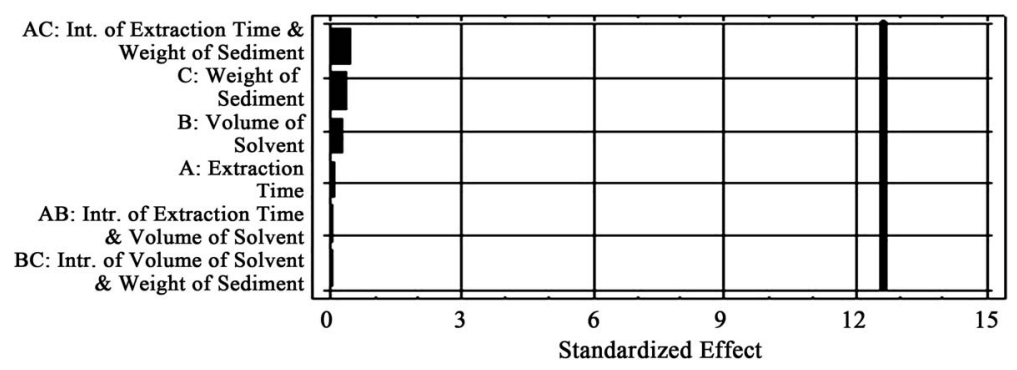

(d)

Figure 2. (a) Pareto chart for sediment HSSPME analysis; (b) Pareto chart for direct SPME water analysis; (c) Pareto chart for soxhlet; (d) Pareto chart for ultrasonic bath. 
Figure 2(b) shows the pareto chart for direct SPME analysis. This graph shows the importance of the factors and their relationships. According to the graph all the factors and interactions were below the line showing the degree of the significance (line at 13 in standardized effects axis). Therefore there was no significantly important factor affecting analysis performance. However the most important factor was adsorption time which was slightly more important than adsorption temperature. The inlet temperature had the least importance. Order of the importance of the factors were; adsorption time $>$ adsorption temperature $>$ interaction of adsorption temperature and adsorption time $>$ inlet temperature $>$ interaction of inlet temperature and adsorption time $>$ interaction of adsorption temperature and inlet temperature.

According to previous soxhlet analysis results DCM/ acetone was found to be best and used during the screening analysis experiments. For the extraction recovery calculations, 3rd surrogates (p-terphenyl D14) responses were used.

According to the standardized pareto chart (Figure 2(c)), sediment amount was the most important. The least important factor was the solvent amount. The importance of the factors and interactions were found as sediment amount $>$ interaction of extraction time and sediment amount $>$ extraction time $>$ interaction of solvent volume and sediment amount $>$ solvent volume $>$ interaction of extraction time and solvent volume. No one of the factors or interactions was significantly important.

As in the case of soxhlet analysis, for ultrasonic bath extraction DCM/acetone (best solvent) was also used as a solvent. Calculated surrogate efficiency $\left(3^{\text {rd }}\right.$ surrogate; p-terphenyl D14) values were used for drawing pareto chart.

According to pareto analysis in Figure 2(d) sediment and time relationship could be investigated and solvent had the less importance. There was no significantly important factor or interaction. The order of the importance was found to be interaction of time and sediment $>$ sediment amount $>$ solvent amount $>$ extraction time $>$ interaction of time and solvent $>$ interaction of solvent and sediment.

\subsection{Response Surface Methodology}

Response surface methodology is a method combining mathematical and statistical techniques based on fitting of a polynomial equation to the experimental data obtained. It describes the behaviour of a data set. In order to use this method several variables that influence the response must exist. The objective is to optimize the levels of these variables simultaneously to obtain the best performance. Firstly experimental design, defining which experiments should be carried out, was chosen and then the response surface methodology was applied [15]. Here, the data obtained from factorial design of each extraction techniques were discussed.

\subsubsection{HSSPME}

Response surface graph for the HSSPME analyses was given in Figure 3(a).

As can be seen from the estimated response surface graph maximum surrogate peak area (average of the peak areas of two surrogates; nitrobenzene D5 and 2-fluorobiphenyl) was observed when factor $\mathrm{A} ; \mathrm{T}_{\text {adsorption }}\left({ }^{\circ} \mathrm{C}\right), \mathrm{B}$; $\mathrm{T}_{\text {inlet }}\left({ }^{\circ} \mathrm{C}\right)$ and $\mathrm{C}$; $\mathrm{t}_{\text {desorption }}$ (hour) were at their maximum values (+1) which were 80,280 and 1 respectively. The lowest recovery value was observed when all factors are $(-1)$; that is when they were at their minimum values.

\subsubsection{Direct SPME}

Surface response analysis for SPME analyses was given in Figure 3 (b).

As can be seen from the estimated response surface graph maximum surrogate peak area (average of the peak areas of 2 surrogates; nitrobenzene D5 and 2-fluorobiphenyl) was observed when Factor A; $\mathrm{T}_{\text {adsorption }}\left({ }^{\circ} \mathrm{C}\right)$ and $\mathrm{B}$; $\mathrm{T}_{\text {inlet }}\left({ }^{\circ} \mathrm{C}\right)$ were at their lowest levels $(-1)$; room temperature and 220 respectively and Factor $\mathrm{C}$; $\mathrm{t}_{\text {desorption }}(\mathrm{min})$ was in its maximum level $(+1) ; 20$. The lowest peak area was found when factor $\mathrm{A}$ and factor $\mathrm{B}(+1)$ and factor $\mathrm{C}$ is $(-1)$.

\subsubsection{Soxhlet Extraction}

Response surface analysis result for soxhlet was shown in Figure 3(c).

For the surrogate recovery calculations, surrogate 3 (2fluorobiphenyl) was used. The maximum surrogate recovery was observed when factor A; time (min), B; solvent $(\mathrm{ml})$ and $\mathrm{C}$; sediment $(\mathrm{g})$ were at their maximum levels $(+1) ; 24,500,50$ respectively. The minimum surrogate recovery was observed when all factors were at their lowest levels $(-1)$.

\subsubsection{Ultrasonic Bath Extraction}

Estimated response surface analysis for ultrasonic bath extraction was given in Figure 3(d).

For the surrogate recovery; surrogate 3 (2-fluorobiphenyl) was used. The maximum was observed when factor A: time $(\mathrm{min})$ and $\mathrm{B}$ : solvent $(\mathrm{ml})$ were at their low level $(-1)$ and factor $\mathrm{C}$ : sediment amount $(\mathrm{g})$ was at its maximum level $(+1)$ : which were $15 \mathrm{~min}, 20 \mathrm{ml}$ and 9 $\mathrm{g}$ respectively. The minimum was observed when factor $\mathrm{A}$ and $\mathrm{B}$ were at their maximum values $(+1)$ and factor $\mathrm{C}$ was in its low value $(-1)$. 


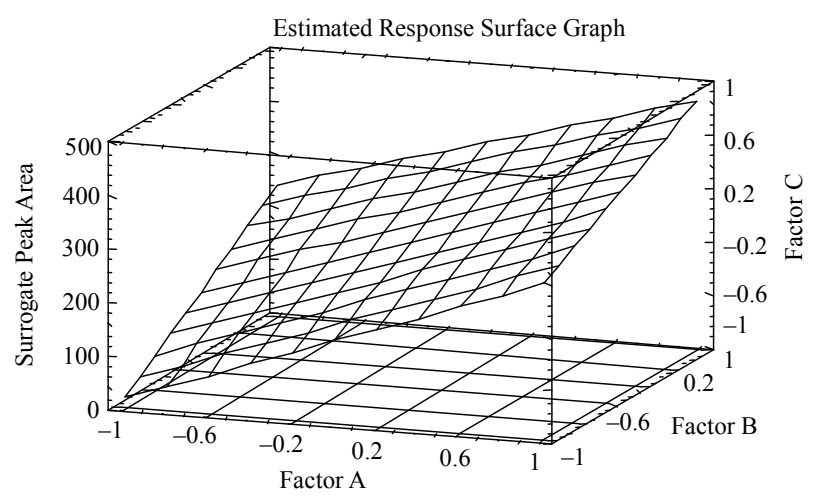

(a)

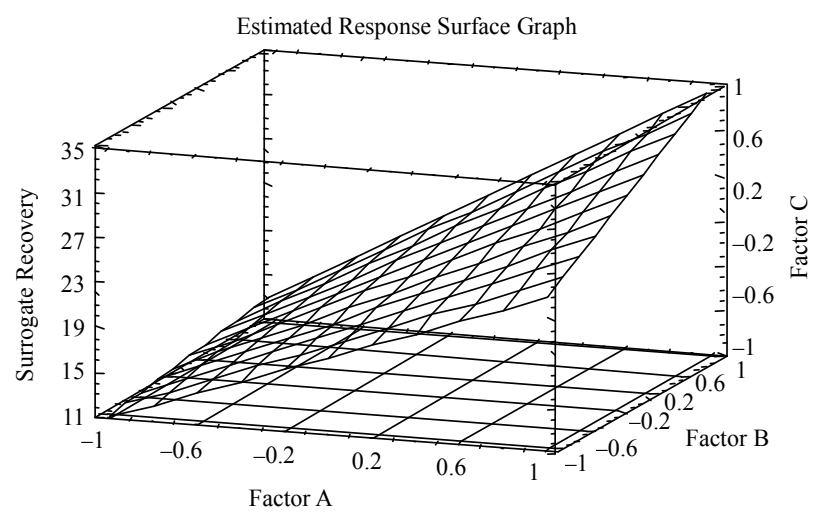

(c)

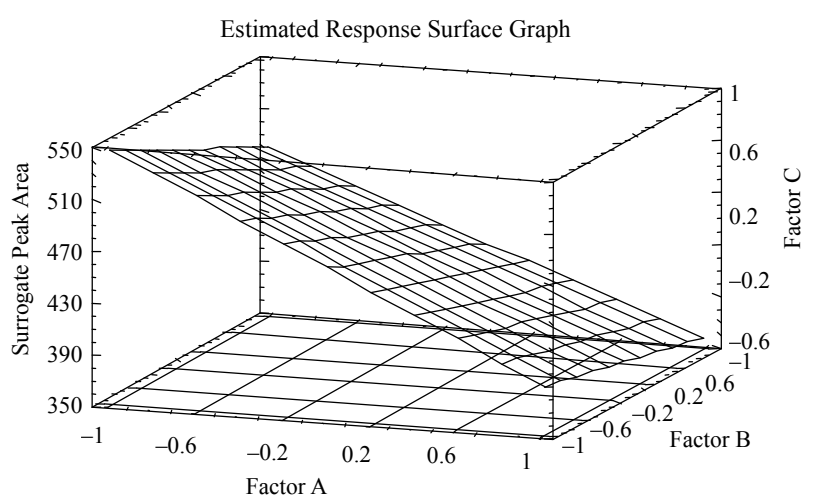

(b)

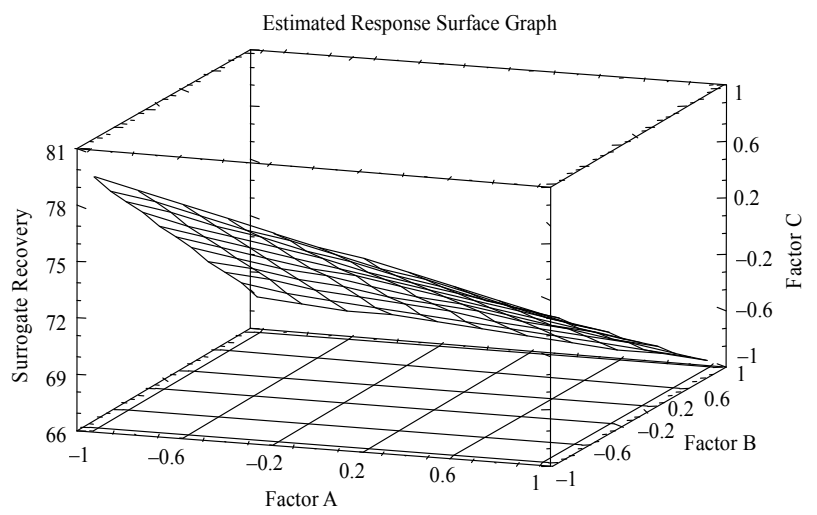

(d)

Figure 3. (a) Response surface for HSSPME sediment analysis; (b) Response surface for direct-SPME; (c) Response surface for soxhlet extraction; (d) Response surface for ultrasonic bath.

\subsection{Influences of the Selected Parameters}

To see the influences of the selected parameters on extraction efficiency, it should be looked at the models created by statistical analysis model created according to design matrix using any software package like SPSS. Positive or negative contribution of the factors and their amounts were easily observed from the models. By using created model it is possible to calculate theoretical extraction efficiency only by putting the value of the factor. The statistical model created according to the design matrix was given in the following Equation (1):

$$
\begin{aligned}
Y= & b_{0}+b_{1} x_{1}+b_{2} x_{2}+b_{3} x_{3} \\
& +b_{12} x_{1} x_{2}+b_{13} x_{1} x_{3}+b_{23} x_{2} x_{3}
\end{aligned}
$$

where $\mathrm{Y}$ is the response variable, $\mathrm{b}_{0}$ is the general mean, $\mathrm{b}_{1}, \mathrm{~b}_{2}, \mathrm{~b}_{3}, \mathrm{~b}_{12}, \mathrm{~b}_{13}, \mathrm{~b}_{23}$ are the factors effect and $\mathrm{x}_{1}, \mathrm{x}_{2}, \mathrm{x}_{3}$ are the factors. Student test with a p-value was used to test the significance of the factors. When p-values of the factors were less than 0.05 , they were considered as statistically significant factors [5].

Discussion related for this model discussed below.

\subsubsection{HSSPME}

For this technique the model formulation is given in Equation 2. As can be seen from the Equation (3), factor $\mathrm{A}, \mathrm{B}$ and $\mathrm{C}$ had positive contribution on the extraction efficiency. Among the factors, the greatest contribution was belong to factor A and smallest contribution was from factor C. Effect of the interactions was also understood from the model. For example interaction of factor $\mathrm{A}$ and $\mathrm{B}$ negatively contributed to the extraction efficiency however interaction of factor $\mathrm{A}$ and $\mathrm{C}$ is positively contributed and the amount of their contributions were nearly the same.

Extraction Efficiency (peak area)

$$
\begin{aligned}
= & 257.875+124.125 \times \text { Factor } \mathrm{A}+100.875 \times \text { Factor } \mathrm{B} \\
& +22.375 \times \text { Factor } \mathrm{C}-13.875 \times \text { Factor } \mathrm{A} \times \text { Factor } \mathrm{B} \\
& +13.625 \times \text { Factor } \mathrm{A} \times \text { Factor } \mathrm{C} .
\end{aligned}
$$

\subsubsection{SPME}

With the same approach the formulation is given in Equation (3). As can be seen from Equation (3) factor A and $\mathrm{B}$ were affecting extraction efficiency negatively. On the other hand factor $\mathrm{C}$ had positive effect on the extrac- 
tion efficiency. According to the model; factor $\mathrm{C}>$ factor $\mathrm{A}>$ factor $\mathrm{B}$ in terms of contribution to the extraction efficiency. Effects of the interactions were also observed from the model. Interaction of A and B was the least important.

Extraction Efficiency (peak area)

$=443.75-70.5 \times$ Factor $\mathrm{A}-24.5 \times$ Factor $\mathrm{B}$

$+74.0 \times$ Factor $\mathrm{C}+9.25 \times$ Factor $\mathrm{A} \times$ Factor $\mathrm{B}$

$-32.25 \times$ Factor $\mathrm{A} \times$ Factor $\mathrm{C}$

$+14.75 \times$ Factor $\mathrm{B} \times$ Factor $\mathrm{C}$.

\subsubsection{Soxhlet Extraction}

Likewise for soxhlet extraction formulation is in the Equation (4), all the factors and the interactions contribute positively and the order of importance was following; factor $\mathrm{C}>$ factor $\mathrm{A}>$ factor $\mathrm{B}$. Interaction of the $\mathrm{A}$ and $\mathrm{B}$ was the least important.

Extraction Efficiency (surrogate recovery)

$=20.955+8.655 \times$ Factor $\mathrm{A}+3.1125 \times$ Factor $\mathrm{B}$

$+12.285 \times$ Factor $\mathrm{C}+1.9025 \times$ Factor $\mathrm{A} \times$ Factor $\mathrm{B}$

$+10.27 \times$ Factor $\mathrm{A} \times$ Factor $\mathrm{C}$

$+3.1125 \times$ Factor B $\times$ Factor $\mathrm{C}$.

\subsubsection{Ultrasonic Bath Extraction}

As can be understood from the Equation (5) all factors contributed negatively and the order of the contribution of the factors were found as; factor $\mathrm{C}>$ factor $\mathrm{B}>$ factor $\mathrm{A}$. Interaction of $\mathrm{A}$ and $\mathrm{C}$ was the most important among them.

Extraction Efficiency (surrogate recovery)

$=71.9375-1.7625 \times$ Factor $\mathrm{A}-4.7875 \times$ Factor $\mathrm{B}$

$-7.1125 \times$ Factor $\mathrm{C}+0.9625 \times$ Factor $\mathrm{A} \times$ Factor $\mathrm{B}$

$-8.2125 \times$ Factor $\mathrm{A} \times$ Factor $\mathrm{C}$

$-0.7375 \times$ Factor $\mathrm{B} \times$ Factor $\mathrm{C}$.

\subsection{Comparison of Extraction Methods}

By using surface response graphs best optimum values were chosen for each extraction methods and methods were compared according to surrogate recovery results. The optimum surrogate recovery value for each method was determined by using the optimized values of factors in the Equations (3)-(6). Two $\mu$ g surrogate was used for both HSSPME and Direct-SPME. In order to calculate $\%$ surrogate recovery of HSSPME, optimum peak area determined from HSSPME, was divided into the optimum peak area determined from direct-SPME and multiplied with 100 as given in the formula below.

$\%$ Surrogate Recovery for HSSPME

$=$ Found Optimum Peak Area of Added Surrogate (HSSPME) *100/Found Optimum Peak
Area of Added Surrogate (Direct-SPME)

In order to calculate \% surrogate recovery of ultrasonic and soxhlet extraction, each concentration (found as a result of each run), was divided into 10 and multiplied with 100 as given below.

$$
\begin{aligned}
& \% \text { Surrogate Recovery for UB and Soxhlet } \\
&= \text { Found Concentration of Surrogate } * 100 / \text { Added } \\
& \text { Concentration of Surrogate }(10 \mathrm{mg} / \mathrm{L})
\end{aligned}
$$

The optimization studies of extraction methods showed that ultrasonic bath extraction was the best in terms of surrogate recovery (81\%) but HSSPME extraction (79\% recovery) was also applicable to extraction of PAHs from sea sediments and it was a good alternative to the ultrasonic bath for the moderately or less polluted samples. Soxhlet had the poorest extraction efficiency $(60 \%$ recovery). Therefore ultrasonic bath extraction method with optimized parameters was used for the analysis of Ölüdeniz sediment samples. The optimized soxhlet extraction system might also be used for the analysis of sediment samples for the less polluted samples.

\subsection{Validation of the Method}

As instrumental analyses, two methodologies namely GC-MS and GC-FID were used in this study and their features were discussed below.

\subsubsection{GC-FID Analysis}

Before starting analysis with GC-FID, some important parameters like injection volume, inlet temperature, carrier gas (He) flow rates, oven temperature program parameters (initial oven temperature, ramp rate and initial time), make-up gas flow rate and flame ionisation detector (FID) temperature were optimised. Peak areas were considered for the comparisons. GC conditions for determination of PAHs were as follows.

After trying different columns, HP-5 5\% Phenyl Methyl Siloxane column (30 m length and $0.32 \mathrm{~mm}$ ID $0.25 \mu \mathrm{m}$ film thickness) having best resolution, higher peak areas and lower background was chosen as best and used during PAH analysis. Helium was used both carrier and make up gas. Splitless mode was used. Injection volume was $2 \mu \mathrm{l}$ and inlet temperature was $300^{\circ} \mathrm{C}$. He flow was $1.5 \mathrm{ml} \cdot \mathrm{min}^{-1}$ similar to the GC-MS conditions. Hydrogen and air ratios were optimized and their values were chosen as $40 \mathrm{ml} \cdot \mathrm{min}^{-1}$ and $400 \mathrm{ml} \cdot \mathrm{min}^{-1}$ respectively. It was necessary to optimize these values to create more stable flame which provides better ionization and lower background. Linear velocity was $27 \mathrm{~cm} \cdot \mathrm{s}^{-1}$ and FID temperature was $325^{\circ} \mathrm{C}$. It was necessary to keep this temperature higher than the oven temperature not to give any damage to detector therefore $300^{\circ} \mathrm{C}$ and $325^{\circ} \mathrm{C}$ were tried 
and according to the optimization studies $325^{\circ} \mathrm{C}$ was chosen as the best. Oven temperature program was $50^{\circ} \mathrm{C}$ at $2 \mathrm{~min}, 8^{\circ} \mathrm{C} / \mathrm{min} 280^{\circ} \mathrm{C}$ at $10 \mathrm{~min}$ (total run $=40.75$ $\min )$.

The method detection limits (MDL) of the each target analytes for GC-FID were determined and the results were given in Table 2.

Each target compound was identified by its retention time of the analyte. The width of the retention time window was 0.01 min to identify the target compounds. The peak areas were integrated by chemstation and manually adjusted if necessary. For controlling the retention times of the analytes, GC-MS was used.

The standard curves were obtained by using 10, 50, 100,500 and $1000 \mu \mathrm{g} \cdot \mathrm{l}^{-1}$ PAH standard and surrogates. The response curves for 16 PAHs were linear with correlation coefficients around 0.99 . The method detection limits ranged from $0.006-0.028 \mathrm{mg} / \mathrm{L}$ as was shown in Table 2.

\subsubsection{GC-MS Analysis}

The GC-MS conditions were the same with GC-FID. In addition to them MS source temperature was $230^{\circ} \mathrm{C}$ and MS quadrupole temperature was $150^{\circ} \mathrm{C}$.

The standard curves were obtained 10, 50, 100, 500 and $1000 \mu \mathrm{g}^{-1} \mathrm{PAH}$ standards and the response curves for 16 PAHs were linear with correlation coefficients around 0.99 . The range of $r$ values lies between 0.997 1.000. The method detection limits ranged from 0.000012 - $0.000390 \mathrm{mg} / \mathrm{L}$ as can be seen from Table 2. The method detection limits were determined based on the concentration (or amount) of an analyte which gave a signal three times the background noise.

\subsubsection{Accuracy Test}

The NIST SRM 1597a reference material was analyzed in order to assess the accuracy of the developed methods (GC-FID and GC-MS). The SRM 1597 offers certified contents for 9 PAHs. Table 3 shows the concentrations and recoveries of PAHs in SRM 1597a $\left(\mathrm{mg} \cdot 1^{-1}\right)$ for GC-MS. As can be seen, $\%$ errors of individual PAH are lying between $5.74 \%$ - 46.6\%. The concentrations and recoveries of PAHs for GC-FID were also shown in Table 3. It can be seen that the percent errors were in the

Table 2. Method detection limits (MDL) of PAHs for GC-FID and GC-MS.

\begin{tabular}{cccc}
\hline No & Name & GC-FID MDL $\left(\mathrm{mg} \cdot \mathrm{L}^{-1}\right)$ & GC-MS MDL $\left(\mathrm{mg} \cdot \mathrm{L}^{-1}\right)$ \\
\hline 1 & Naphthalene & 0.0110 & 0.0000980 \\
2 & Acenaphtylene & 0.00700 & 0.0000560 \\
3 & Acenaphtene & 0.0160 & 0.0000250 \\
4 & Fluorene & 0.00900 & 0.0000370 \\
5 & Phenanthrene & 0.0140 & 0.0000390 \\
6 & Anthracene & 0.0100 & 0.0000250 \\
7 & Fluoranthene & 0.00600 & 0.0000240 \\
8 & Pyrene & 0.0130 & 0.0000120 \\
9 & Benzo $(a)$ anthracene & 0.0140 & 0.0000490 \\
10 & Chrysene & 0.0140 & 0.000215 \\
11 & Benzo $(b)$ fluoranthene & 0.00900 & 0.000124 \\
12 & Benzo $(k)$ fluoranthene & 0.0160 & 0.0000720 \\
13 & Benzo $(a)$ pyrene & 0.0190 & 0.0000740 \\
14 & Indeno $(1,2,3-c d)$ pyrene & 0.0280 & 0.000193 \\
15 & Dibenzo $(a, h)$ anthracene & 0.0140 & 0.000390 \\
16 & Benzo $(g, h, i)$ perylene & 0.0140 & 0.000164 \\
\hline
\end{tabular}

Table 3. Certified concentrations and \% errors of PAHs in SRM 1597a $\left(\mathrm{mg} \cdot \mathrm{l}^{-1}\right)$ for GC-MS $(\mathrm{n}=4)$.

\begin{tabular}{cccc}
\hline SRM 1597a & Certified Conc. (mg/L) & GC-FID \% Error & GC-MS \% Error \\
\hline Nap & $1000 \pm 50.0$ & 1.70 & -46.6 \\
Phe & $400 \pm 4.00$ & 25.0 & -17.8 \\
Fla & $278 \pm 4.00$ & 2.10 & -9.67 \\
Pyr & $204 \pm 3.00$ & 13.0 & -5.74 \\
BaA & $85.3 \pm 3.40$ & 4.80 & 23.5 \\
Chr & $62.0 \pm 1.00$ & 20.0 & 32.7 \\
BaP & $82.9 \pm 5.30$ & -1.40 & -46.9 \\
IcP & $52.1 \pm 4.00$ & 8.20 & -13.5 \\
BgP & $46.5 \pm 6.70$ & -20.0 & -23.7 \\
\hline
\end{tabular}


range of $1.40 \%-25.0 \%$, demonstrating a good quantitative agreement and a satisfactory performance of the method. It can be understood from the Table 3 that; GC-FID was better than GC-MS in terms of accuracy.

\subsubsection{Application of Method to SRM 1941b}

Since the comparison of extraction methods showed that best extraction method was ultrasonic bath extraction, SRM 1941b was dissolved by using this method. The best optimum conditions for ultrasonic bath extraction obtained from response from chart (Figure 3(d)). Table 4 shows the recovery values and percent errors for the PAHs in SRM 1941b. As can be seen recovery values are very good and changing between $67 \%-101 \%$.

\subsection{Application of the Method to Sediment Samples}

Since analysis of the Ölüdeniz sediment samples were done before the optimization of the extraction methods, ultrasonic bath method based on EPA 3550 B [13] was used for the extraction of 5 sediment samples. Solvents were also selected according to the EPA $3550 \mathrm{~B}$ method and hexane-DCM (1:1) pair was chosen as a solvent.

The extraction efficiency was calculated by using surrogate spike containing 2-Fluorobiphenyl in DCM and it was found as $91 \%$. The procedure for the extraction of sediment was summarized below.

Five gram (air dried) sediment was weighted into an extraction vessel. After spiking with surrogate standard, $1 \mathrm{ml}$ of $6.5 \mu \mathrm{g} / \mathrm{ml}$ 2-fluorobiphenyl in methylene chloride, the sample was extracted in a $50 \mathrm{ml}$ beaker for about 10 minutes with an $10 \mathrm{ml}$ of DCM and hexane mixture $(1: 1$, $\mathrm{v} / \mathrm{v})$. The same procedure was repeated with a mixture of hexane $(5 \mathrm{~mL})$ and DCM $(5 \mathrm{~mL})$ twice more for a total volume of $30 \mathrm{ml}$.

After getting the extract, it was first transferred to the glass wool column to get rid of the impurities in the solution. Secondly, the extract was eluted from sodium sulphate column with the use of pasteur pipette. Sodium sulphate column was $20 \mathrm{~cm}$ in length, plugged with glass wool and pre-washed twice with hexane and dichloromethane.

After drying, extracts were preconcentrated under the stream of nitrogen and volume was reduced to $1 \mathrm{ml}$ and the final solution was kept in a $2 \mathrm{ml}$ amber glass vials tapped with a teflon-lined cap and preserved in a refrigerator at $4^{\circ} \mathrm{C}$ until the analysis with GC-FID. The quantification was made against an external standard analytical curve, constructed with solutions prepared from the Supelco PAH standard in DCM: methanol (1:1).

In order to make a precision comparison between $\mathrm{GC}$ MS and GC-FID, three selected sediments among five extracted sediments, were analyzed with both GC-FID and GC-MS at the same time, with similar conditions. By considering accuracy and precision values of the methods, best analysis method was chosen.

Table 5 shows the comparison of mean concentrations of 5 analyzed sediment samples and their precisions.

\subsection{Literature Comparison}

Different extraction methods (soxhlet, pressurized fluid extraction, shaking, microwave and SPME) and instruments (GC-MS, GC-FID, HPLC-DAD) were compared in terms of \% recovery in Table 6 [14]. Optimized ultrasonic bath extraction method was used in this study. This method includes extraction of $9 \mathrm{~g}$ wet sediment sample with $20 \mathrm{ml}$ DCM-Acetone $(1: 1 \mathrm{v} / \mathrm{v})$ for $15 \mathrm{~min}$ utes. These optimum values were obtained from response surface chart. Analysis with GC-FID resulted $81 \%$ recovery with surrogate; 2-Fluorobiphenyl. Recoveries obtained for GC-MS; $41 \%$ for Acenaphtene D10, 60\% for Phenanthrene D10, 85\% for Chrysene D12 and 86\% for Perylene D12. As can be seen from \% recoveries in Table 6, recovery values for this study are quite high or comparable with the other values.

Table 4. SRM 1941b analysis results.

\begin{tabular}{lcccc}
\hline & Certified Conc. (ug/kg) & Found Conc. (ug/kg) & \% Error & \% Recovery \\
\hline Naphthalene & $848 \pm 95.0$ & $859 \pm 33.0$ & 1.00 & 101 \\
Fluorene & $85.0 \pm 15.0$ & $73.0 \pm 13.0$ & -14.0 & 86.0 \\
Phenanthrene & $406 \pm 44.0$ & $333 \pm 23.0$ & -18.0 & 82.0 \\
Pyrene & $581 \pm 39.0$ & $386 \pm 27.0$ & -33.0 & 67.0 \\
Benzo(a)anthracene & $335 \pm 25.0$ & $225 \pm 24.0$ & -33.0 & 67.0 \\
Benzo(b)fluoranthene & $453 \pm 21.0$ & $344 \pm 48.0$ & -24.0 & 76.0 \\
Benzo(e)pyrene & $325 \pm 25.0$ & $351 \pm 52.0$ & 8.00 & 108 \\
Benzo(a)pyrene & $358 \pm 17.0$ & $330 \pm 22.0$ & -8.00 & 92.0 \\
Benzo(g,h,i) perylene & $307 \pm 45.0$ & $267 \pm 32.0$ & -13.0 & 87.0 \\
Indeno (1,2,3-c,d) pyrene & $341 \pm 57.0$ & $333 \pm 79.0$ & -2.00 & 98.0 \\
\hline
\end{tabular}


Table 5. Comparison of the precisions of GC-MS and GC-FID measurements $(n=5)$.

\begin{tabular}{ccccc}
\hline & GC-MS & GC-MS & GC-FID & GC-FID \\
\hline PAHs & $\begin{array}{c}\text { Mean Conc. } \\
(\mathrm{mg} / \mathrm{kg})\end{array}$ & $\begin{array}{c}\text { Precision } \\
\text { Std dev. }\end{array}$ & $\begin{array}{c}\text { Mean Conc. } \\
(\mathrm{mg} / \mathrm{kg})\end{array}$ & $\begin{array}{c}\text { Precision } \\
\text { Std dev. }\end{array}$ \\
Nap & 0.0177 & 0.00410 & $\mathrm{ND}$ & ND \\
Acy & 0.0370 & 0.0124 & $\mathrm{ND}$ & ND \\
Ace & 0.0347 & 0.00790 & 0.662 & 0.480 \\
Fle & 0.0101 & 0.00440 & 0.0132 & 0.00860 \\
Phe & 0.406 & 0.517 & 0.439 & 0.380 \\
Ant & 0.114 & 0.0730 & 0.0405 & 0.0583 \\
Fla & 0.0960 & 0.141 & 0.248 & 0.338 \\
Pyr & 0.160 & 0.223 & 0.207 & 0.249 \\
BaA & 0.393 & 0.602 & 0.182 & 0.232 \\
Chr & 0.0830 & 0.115 & 0.291 & 0.250 \\
BbF & 0.104 & 0.131 & 0.0144 & 0.0147 \\
BkF & 0.0759 & 0.0898 & 0.0231 & 0.0239 \\
BaP & 0.0516 & 0.0679 & 0.178 & ND \\
IcP & 0.271 & 0.341 & 0.416 & ND \\
DaA & 0.0268 & 0.0242 & 0.0634 & 0.0559 \\
BgP & 0.0740 & 0.0744 & 0.0776 & 0.0655 \\
\hline
\end{tabular}

Table 6. Literature comparison.

\begin{tabular}{|c|c|c|c|}
\hline Sample Preparation & Instrument & \% Recovery & References \\
\hline Soxhlet extraction with dichloromethane-16h. & GC-MS & $90-118$ & {$[15]$} \\
\hline $\begin{array}{l}\text { Pressurized solvent extraction with a mixture } \\
\text { of dichloromethane and acetone }(3: 1, \mathrm{v} / \mathrm{v}) \text {. }\end{array}$ & GC-MS & $75-105$ & {$[16]$} \\
\hline $\begin{array}{l}\text { Soxhlet extraction for } 20 \mathrm{~h} \text { with } \\
\text { Dichloromethane. }\end{array}$ & GC-MS & $90-105$ & {$[17]$} \\
\hline Ultrasonication by acetonitrile $-15 \mathrm{~min}$ & $\begin{array}{l}\text { GC-MS } \\
\text { HPLC-DAD }\end{array}$ & $\begin{array}{l}\text { GC-MS: } 53-73 \text {, } \\
\text { HPLC-DAD: } 64-92\end{array}$ & {$[18]$} \\
\hline $\begin{array}{l}\text { A. Soxhlet extraction: with a solution }(1: 1 \mathrm{v} / \mathrm{v}) \\
\text { n-hexane-acetone B. Supercritical fluid } \\
\text { extraction: with co-solvents: n-hexane, } \\
\text { methanol and toluene. }\end{array}$ & GC-MS & A: $73.8-89.1 \mathrm{~B}:>90$ & {$[19]$} \\
\hline Shaking with dichloromethane for $30 \mathrm{~min}$. & HPLC-DAD & $>80$ & {$[20]$} \\
\hline $\begin{array}{l}\text { Microwave extraction followed by solid phase } \\
\text { microextraction (with PDMS and PA fiber). }\end{array}$ & GC-MS & $58.6-112$ & {$[21]$} \\
\hline $\begin{array}{l}\text { Ultrasonication for } 15 \mathrm{~min} \text { with } \mathrm{DCM} / \text { Acetone } \\
(1: 1 \mathrm{v} / \mathrm{v}) \text {. }\end{array}$ & $\begin{array}{l}\text { GC-FID } \\
\text { GC-MS }\end{array}$ & $\begin{array}{l}\text { GC-FID: }>81 \\
\text { GC-MS: } 41-86\end{array}$ & This study \\
\hline
\end{tabular}

\section{Conclusions}

Different extraction methodologies were optimised for sixteen PAH compounds in sediment samples. Sixteen PAH compounds in sediment samples were determined with high recoveries by using HP 6890 series GC coupled to a flame ionization detector. The optimization studies of extraction methods showed that ultrasonic bath ex- traction was the best in terms of surrogate recovery but SPME extraction was also suitable for the extraction of PAHs from sea sediments. The detection limits obtained were comparable with the ones in EC directives.

It was also found that, optimized soxhlet extraction system may also be used for the analysis of less polluted sediment samples. For the analysis of naphthalene and acenaphtylene which were below detection limit of GC- 
FID, GC-MS was offered as a better alternative.

Concentrations of PAHs in sediments were generally moderate in Oludeniz sediment samples. Acenaphtene and chrysene were dominant ones among the sixteen PAHs analysed and their concentrations were 0.620 and $0.515 \mathrm{mg} \cdot \mathrm{kg}^{-1}$ relatively.

\section{References}

[1] J. Xu, Y. Yu, P. Wang, W. Guo, S. Dai and H. Sun, "Polycyclic Aromatic Hydrocarbons in the Surface Sediments from Yellow River, China," Chemosphere, Vol. 67, No. 7, 2007, pp. 1408-1414. doi:10.1016/j.chemosphere.2006.10.074

[2] L. Culotta, C. D. Stefano, A. Gianguzza, M. R. Mannino and S. Orecchio, "The PAH Composition of Surface Sedi- ments from Stagnone Coastal Lagoon, Marsala (Italy)," Marine Chemistry, Vol. 99, No. 1-4, 2006, pp. 117-127. doi:10.1016/j.marchem.2005.05.010

[3] Y. F. Song, X. Jing, S. Fleischmann and B. M. Wilke, "Comparative Study of Extraction Methods for the Determination of PAHs from Contaminated Soils and Sediments," Chemosphere, Vol. 48, No. 9, 2002, pp. 9931001. doi:10.1016/S0045-6535(02)00180-7

[4] N. F. Leite, P. P. Zamora and M. T. Grassi, "Multifactorial Optimization Approach for the Determination of Polycyclic Aromatic Hydrocarbons in River Sediments by Gas Chromatography-Quadrupole İon Trap Selected İon Storage Mass Spectrometry," Journal of Chromatography A, Vol. 1192, No. 2, 2008, pp. 273-281. doi:10.1016/j.chroma.2008.03.067

[5] M. B Deschamps, J. J. Daudin and E. Barriuso, “An Experimental Design Approach to Optimise the Determination of Polycyclic Aromatic Hydrocarbons from Rainfall Water Using Stir Bar Sorptive Extraction and High Performance Liquid Chromatography-Fluorescence Detection," Journal of Chromatography A, Vol. 1167, No. 2, 2007, pp. 143-153. doi:10.1016/j.chroma.2007.08.025

[6] C. R. T. E. Tarley, G. Silveira, W. N. L. Santos, G. D. Matos, E. G. P. Silva, M. A. Bezerra, M. Miró and S. L. C. Ferreira, "Chemometric Tools in Electro Analytical Chemistry: Methods for Optimization Based on Factorial Design and Response Surface Methodology," Micro chemical Journal, Vol. 92, No. 1, 2009, pp. 58-67. doi:10.1016/j.microc.2009.02.002

[7] J. N. Miller and J. C. Miller, "Statistics and Chemometrics for Analytical Chemistry," 4th Edition, Pearson Education, England, 2000, Chapter 7.

[8] B. E. Berg, H. S. Lund, A. Kringstad and A. L. Kvernheim, "Routine Analysis of Hydrocarbons, PCB and PAH in Marine Sediments Using Supercritical $\mathrm{CO}_{2}$ Extraction," Chemosphere, Vol. 38, No. 3, 1999, pp. 587-599. doi:10.1016/S0045-6535(98)00213-6

[9] D. R. Banjoo and P. K. Nelson, "Improved Ultrasonic Extraction Procedure for the Determination of Polycyclic Aromatic Hydrocarbons in Sediments," Journal of Chro- matography A, Vol. 1066, No. 1-2, 2005, pp. 9-18. doi:10.1016/j.chroma.2005.01.033

[10] H. Budzinski, M. Letellier, P. Garrigues and K. Le Menach, "Optimisation of the Microwave-Assisted Extraction in Open Cell of Polycyclic Aromatic Hydrocarbons from Soils and Sediments: Study of Moisture Effect," Journal of Chromatography A, Vol. 837, No. 1-2, 1999, pp. $187-$ 200. doi:10.1016/S0021-9673(99)00067-9

[11] EPA METHOD 3540 C, 1996.

[12] EPA METHOD SW-846 3550C, 2007.

[13] EPA METHOD 3550 B, 1996.

[14] A. Nikolaou, M. Kostopoulou, G. Lofrano and S. Meric, "Determination of PAHs in Marine Sediments: Analytical Methods and Environmental Concerns," Global Nest Journal, Vol. 11, 2009, pp. 391-405.

[15] C. Anyakora, A. Ogbeche, P. Palmer, H. Coker, G. Ukpo and C. Ogah, "GC/MS Analysis of Polynuclear Aromatic Hydrocarbons in Sediment Samples from the Niger Delta Region," Chemosphere, Vol. 60, No. 7, 2005, pp. 990997. doi:10.1016/j.chemosphere.2004.12.073

[16] R. Boonyatumanond, G. Wattayakorn, A. Togo and H. Takada, "Distribution and Origins of Polycyclic Aromatic Hydrocarbons (PAHs) in Riverine, Estuarine and Marine Sediments in Thailand", Marine Pollution Bulletin, Vol. 52, No. 8, 2006, pp. 942-956. doi:10.1016/j.marpolbul.2005.12.015

[17] C. H. Koch, J. S. Khim, D. L. Villeneuve , K. Kannan and J. P. Giesy, "Characterization of Trace Organic Contaminants in Marine Sediment from Yeongil Bay, Korea: 1. Instrumental Analyses," Environmental Pollution, Vol. 142 , No. 1,2006 , pp. 39-47. doi:10.1016/i.envpol.2005.09.005

[18] A. Filipkowska, L. Lubecki and G. Kowalewska, "Polycyclic Aromatic Hydrocarbon Analysis in Different Matri- ces of the Marine Environment," Analytica Chimica Acta, Vol. 547, No. 2, 2005, pp. 243-254. doi:10.1016/j.aca.2005.05.023

[19] V. Librando, O. Hutzinger, G. Tringali and M. Aresta, "Supercritical Fluid Extraction of Polycyclic Aromatic Hy- drocarbons from Marine Sediments and Soil Samples," Chemosphere, Vol. 54, No. 8, 2004, pp. 1189-1197. doi:10.1016/j.chemosphere.2003.07.008

[20] R. A. Gimeno, E. Comas, R. M. Marcé, J. Ferré, F. X. Rius and F. Borrull, "Second-Order Bilinear Calibration for De- termining Polycyclic Aromatic Compounds in Marine Sediments by Solvent Extraction and Liquid Chromatography with Diode-Array Detection," Analytica Chimica Acta, Vol. 498, No. 1-2, 2003, pp. 47-53. doi:10.1016/j.aca.2003.08.057

[21] V. Pino, J. H. Ayala, A. M. Afonso and V. Gonzalez, "Micellar Microwave-Assisted Extraction Combined with Solid-Phase Microextraction for the Determination of Polycyclic Aromatic Hydrocarbons in a Certified Marine Sediment," Analytica Chimica Acta, Vol. 477, No. 1, 2003, pp. 81-91. doi:10.1016/S0003-2670(02)01410-1 